\title{
What Gravity Is: Alzofon-Ionescu Theory beyond Newton, Einstein, Quantum/Entropy Gravity, etc. A Virequest Project Proposal
}

\author{
Lucian Miti Ionescu \\ Department of Mathematics, Illinois State University, Normal, USA \\ Email: lmiones@ilstu.edu
}

How to cite this paper: Ionescu, L.M. (2021) What Gravity Is: Alzofon-Ionescu Theory beyond Newton, Einstein, Quantum/Entropy Gravity, etc. A Virequest Project Proposal. Journal of High Energy Physics, Gravitation and Cosmology, 7, 1182-1189.

https://doi.org/10.4236/jhepgc.2021.73069

Received: June 5, 2021

Accepted: July 23, 2021

Published: July 26, 2021

Copyright $\odot 2021$ by author(s) and Scientific Research Publishing Inc. This work is licensed under the Creative Commons Attribution International License (CC BY 4.0).

http://creativecommons.org/licenses/by/4.0/

\begin{abstract}
Gravity is not a fundamental force; in a nut-shell, it is the result of a noncommutative interaction of the "electric" (i.e. Coulomb type) due to fractional charges of the proton and neutron in $U(2)$-gauge theory, but which have no structure in $U(1)$-gauge theory, being neutral in itself (neutron), or when compensated by the electronic cloud (proton). This is no longer true at the $S U$ (2) Electroweak Theory level, once spherical 3D-symmetry is broken to a finite Platonic group of symmetry within it $\Gamma \rightarrow S U(2)$. The fine splitting of energy levels due to the quark structure (frame basis in $S U(2)$ ) of the electric charge can be experimentally controlled using a MASER to invert the population and orient the nuclei the right way to reduce and turn off Gravity.
\end{abstract}

\section{Keywords}

Quantum Gravity, String Theory, Standard Model

\section{Point, String... Sphere!}

Point-wise Physics, even quantized, cannot rival String Theory, with its breakthrough idea: "blow-up the point into a string!" Physics then evolved naturally to the next "blow-up" when Feynman started to talk about Quantum Mechanics as Quantum Computing: the Block sphere models the 3D frame and Einstein's local time is related to the quantum phase, as Feynman also said: "each particle carries a clock... $\exp (i \omega t)$ " (something to this effect).

....and then a Poincare-Montonen-Olive duality does the rest! Points are dual to spheres (Gauss Charges and EM via relativity) and strings couple with strings 
(Gauss Link and Abrikosov's fluxons).

But Gravity cannot emerge yet, unless we quantize a 3-rd time: from a continuous quantum phase/qubit ( $U(1)$ embedded in $S U(2)$ ), to a finite phase $Z / n$ within the finite qubit (Platonic solids and excitations).

\section{On 20th Ctry Physics}

\subsection{Some Historic Origins}

Tracing its history, Newton's Gravity is the unique "free-flow" model (harmonic, Laplace/Poison, underlying its conformal character) in his general Diff.-Eq. framework of Mechanics, again unique in view of the Hamiltonian version (Poisson, rather than symplectic; again unique in view of the QM/QC ultimate formalism based on Hopf fibration/qubits).

Einstein's General Relativity is a similar general framework for classical physics, based on Differential Geometry, rather than Euclidean model. In fact, Newton's Mechanics models curved motion in flat space, while Einstein's, models "flat motion" (geodesics) in curved space-time (relativistic: "pre-quantum era"); see [1] p.4, etc. It should be stressed that GR is not a model of Gravity like Newton's, but rather a general framework: there is no prescription for the energymomentum tensor that would correspond to the constitutive part of the model; it accommodates Gravity nicely though, incorporating Mach's principles, as explained below.

\subsection{From Classical to Quantum}

The evolution from General Relativity and QFT to Standard Model, GUTs and TOEs will be briefly mentioned.

In brief:

1) General Relativity is a framework for dynamics, in the spirit of Mach's philosophy, introducing for the first time in classical theory the idea of deformations, via (symbolically):

$$
R_{i j}=\frac{1}{2} R g_{i j}+\chi T_{i j}, \quad \text { Ric_Curvature }=\text { Standard_Metric }+ \text { “ } \hbar ” \text { Matter_Tensor. }
$$

It can be explained how perturbing the Green function in QFT, is dual to perturbing the metric, via the principal symbol of the corresponding Laplacian...

Hence QFT in fact largely extends General Relativity at a conceptual level, using a modern mathematical framework, interaction oriented (via propagators), rather then Differential Geometric oriented framework (metric); not to mention the advance through the introduction of the concept of Network via Feynman Diagrams, and later on Quark Diagrams in SM.

Weyl's further Differential Framework of Gauge Theory sets Noether's use of symmetries at the core of the theory, again "upgrading" the classical EinsteinGrossman approach.

Classical QFT a la Weinberg (Pointwise Quantum Mechanics) still makes use of Space-Time, but Feynman's diagrams approach is similar in vein to Einsete- 
nin's emphasis on matter vs. space-time as a coordinate-like receptacle of the Dynamics.

Feynman's interpretation of Quantum Mechanics as Quantum Computing set the dawn of the modern paradigm based on Information: Classical and Quantum.

2) Kaluza-Klein Theory and similar UFTs, are still classical theories. These theories cannot capture the essence of reality: discreetness via quantization of matter, action or information.

In the author's opinion, Gravity is a direct byproduct of this latter discreetness (finite gauge groups), combined with the ensuing anisotropy of the building blocks: proton/neutron. This leads directly to the quantization of angular momentum, as a consequence, rather then a postulate via Quantum Mechanics framework.

3) GUTs and TOEs focus on mathematical aspects, rather than physics foundations. Nevertheless they correctly "guess" the importance of the exceptional Lie groups $E_{6}, E_{7}, E_{8}$ : these are the dynamical counterparts of the binary 3D-point groups (Platonic symmetries), which are the corresponding Weyl groups for the corresponding root systems [2].

4) The breakthrough: "Quantize everything", not just action, but also the $q u$ bit space, i.e. $S U(2)$. This includes quantizing the quantum space and more notably, RESULTS in the quantization of angular momentum! As a consequence, the directions of interactions between systems, i.e. the connection will take discrete values: the emergent Space-Time itself, becomes quantized at the level of symplectic/Hamiltonian formulation (L.I.: use [3] to "bind" $S U(2)$ and $S U(3)$ as it's in the Hopf bundle formalism, as the symmetries of the "qubit").

Quantum Physics's traditional approach is via quantization as a technique starting from Classical Physics aiming towards a Quantum Theory: Dirac's prescription, Heisenberg's CCR, deformation quantization, perturbation theory, etc.

What was needed, is a Quantum Theory "designed from scratch" (basic axioms), not as an "upgrade" of a classical framework via, say Dirac's prescription or some other perturbation approach.

Such a theory is Quantum Computing as the foundation for ClassicalQuantum Logic, together with Quantum Information Dynamics, including the Qubit Model. The idea behind the qubit with its two basic states dates from ancient Tao (Yin-Yang duality), and is present in some modern theories advocating two basic constituents $(\mathrm{T} / \mathrm{N})$.

The later (QC) corresponds and "corrects" the Quark Model of the Standard Model [4]. Quarks constitute a frame in qubit space ( $C^{2}$ is iso to quaternionic space $H$ or $2+2=3+1)$, and are not free particles, hence there is no "strong force" (except as a Theory).

The weak decays can be modeled as transitions via a recoupling theory, so there is no "weak force" either. But the Electroweak Theory is remarkably successful in explaining the experimental data...

5) Why the Electroweak Theory "missed Gravity"? Just because physicists use 
the excuse that Gravity is "too weak to account for measurable effects in Elementary Particle Physics”...But conceptually, it is crucial, because...it just does exist!

An analysis of Electroweak Theory reveals that the "independent" gauge groups $U(1), S U(2)$ and $S U(3)$ can be easily unified by recognizing the symmetries of the Hopf fibration, the central concept of Qubit Model (To be explained-if needed). Then the consequence of finiteness of the gauge group $\Gamma \rightarrow S U(2)$ leads to anisotropy of interactions and a departure from the usual Coulomb type of interaction (Green function), via the structure of charge in baryons (fractional charges are just eigenvalues of operators involved in Gell-Mann Nishijima formula: Lie generators of the groups from the Hopf fibration).

\section{VI Request Project Contributions}

VI theoretical, umbrella contribution, comes from a modern viewpoint based on the Quantum Information new paradigm [5] and a holistic viewpoint of the reality modeled by Quantum Physics [6] [7].

\subsection{Unifying Carriers and Sources: The Network Approach}

The later includes a unification of fermions and bosons, not via supersymmetry of particle or strings physics, but rather as a natural approach via categorification: particles as objects (e.g. baryons as qubits and atoms as quantum registers) and morphisms as channels of communication of quantum information, a modern model of the traditional interactions. This is closely related to Feynman diagrams in QFT, Riemann surfaces in String Theory and the so-called quark lines diagrams in The Standard Model.

The particle-wave duality acquires a natural interpretation in the context of the Network Model approach to interactions.

\subsection{Pointwise Electrodynamics Is Not Enough}

The role of high electric potentials is certainly intriguing. Positive effects when comes to non-conventional methods of propulsion were achieved for instance in Townsend Brown's experiments; they involve rotation, hence associated magnetic effects which represent a form of "curvature".

The Kaluza-Klein approach to modeling Gravity ${ }^{1}$ has no "constitutive force" component, i.e. no justification of why would Gravity emerge or be present in the first place. Like Einstein's Theory, it does not explain Gravity; it just models what we already know about it, usually "author dependent".

The negative result for static configurations is expected, as reported by the HRI experiments.

Remark 3.1 The presence of coherent states in supraconductivity experiments is expected to allow for unusual effects as in Podkletnov's experiments. nevertheless there is a need for an active pumping of lowest energy states into a fine ${ }^{1}$ The use of a 5D-Space-Time is from a modern perspective obsolete; the fiber bundle approach with $U(1)$ fiber is much more versatile. 
splitting states that do not exhibit gravitational pull; and these were not understood enough to be achieved in combination with superconductive states.

\subsection{From an Effective Theory of Gravity to Foundations}

1) VI research sets a modern foundation for F. Alzofon's theory on Gravity modification, which is currently based on a generic thermodynamic formalism and an analogy with adiabatic cooling (TBA).

At a practical, experimental level, VI approach is based on a mechanism of inversion of population of coherent states, characteristic of masers:

- Using coherent states (resonant cavities, LASERS, etc.);

- Using sources of microwaves (a.k.a. torsion waves as generated by transponders, e.g. at the exit of stores).

From a theoretical viewpoint, these states and their energy levels are modeled in terms of finite groups of symmetry ("vertical gauge groups"/crystallographic groups), approach which leads to Platonic groups of symmetry and exceptional Lie algebras for the corresponding dynamics [2].

Further other directions of investigation will be proposed elsewhere... (see [8]).

2) VI proposes an experimental test of Gravitational Induction, corresponding to rotating masses, and analog of the magnetic field in EM (details later on).

This is the result of relativistic formulation of Newtonian theory of Gravity to be implemented in the implementation phase of the collaboration as follows. The conceptual foundations of Newton's Theory of Gravity, consisting of the general DE framework $m a=F_{G}, F_{G}=k m m^{\prime} / r^{2}$, based on Galilei transformations can be implemented in the relativistic framework, by using Lorentz transformations, yielding a Lorentz force type of equation; this will yield the gravitational analog of magnetic field [9] [10].

Besides the scientific interest itself, it is needed to understand the coupling between EM and Gravity (rotating masses), as exhibited by John Searl Generator experiments.

\section{How to Control Gravity and Inertial Mass}

\subsection{What Are the Three "Generations"}

The main system of interest when comes to Gravity, is the nucleus, traditionally modeled as made of neutrons and protons, although these form rather a complex network.

As basic, individual constituents, the "free" neutron, not correlated to a system, should be modeled as the Hopf bundle $S U(2) \rightarrow S^{2}$ (continuous, undetermined orientation); also the negative and positive fractional charges are not yet expressed via a break of symmetry.

The proton-electron system, on the other hand, corresponds to the extension $U(1) \rightarrow S U(2)$; plus brack of symmetry due to finite groups.

When measured (e.g. in a magnetic field), such individual quantum systems 
(neutron/proton) acquire a basis of vectors corresponding to quarks.

In brief, the Platonic finite configurations define the type of constituent quarks.

1) The tetrahedron $T_{4}$ corresponds to $u$ and $d$, self-dual if it would not be for the $n \rightarrow p$ break of symmetry aluded to above;

2) Cube and its dual Octahedron, correspond to $c$ and $s$;

3) Icosahedron and Dodecahedron configurations correspond to what we call top and bottom quarks, as constituents of a baryon.

Therefore there are "3-types" of "flavors" (not 6), band in duality, not pairs organized as "generations" (quark mass, static or dynamic, and theory dependent defined, are significantly different).

The "mixed" combinations, e.g. usb, in Gell-Mann's $S U(3)$-theory, should have models in the finite groups reps theory (like modes of orbitals in electron's theory).

\subsection{Consequences: Gravity Control}

We are primarily concerned with the basic states $n=u d d$ and $p=u u d$, as modeled in the $S U(3)$-theory.

Now both are tetrahedral with distinct charge distribution; the "bound" neutron is in first approximation neutral --+ , while the proton -++ , where the + is twice the opposite of -. Both fractional charges are eigenvalues of a "tensorial Coulomb Law" (non-commutative; Gaussian link integrals etc.).

Both $n$ and $p+e^{-}$are not antipodal symmetric, hence there is ultra-fine split of energy levels in this NC-Coulomb Law that accounts for a preferred orientation exhibiting a tinny attraction we call Gravity.

\subsubsection{Alzofon's Effective Theory}

The thermal chaotic motion yields a distribution of states which invites to an entropic approach to Gravity; the relation to Blackhole thermodynamics laws and Verlinde's Entropic Gravity should be investigated.

But this thermodynamic model of Gravity was clearly described by Frederick Alzofon in the 60s-80s [11] [12]. The quark structure of fractional electric charges and anisotropy due to Platonic symmetry sets its foundations in an "extension" of the Standard Model ("3-rd quantization": that of the gauge group/qubit and quantum phase).

\subsubsection{Dynamic Nuclear Orientation and Microwave Pumping}

Recall that in the finite gauge SM Gravity is always attractive due to the natural orientation of nucleon pairs in the lower energy state of a pair.

A dynamical orientation of the nucleon's spin [13] [14] [15], via orbit-spin interaction can be achieved with a mechanism similar to that of a MASER, pumping energy and inverting the population of such states, in the context of an adequate resonant cavity setup [16].

In the author's opinion, Alzofon's experiment lacked the resonant cavity aspects, which acts as a filter of such inversions. 
The role of the so-called structural cavity effect (SCE) is not understood at this point; it may be of conceptual (and practical) interest in the above DNO process.

\subsection{Controlling Inertial Mass}

The Higgs mechanism has further applications, allowing to "juggle" with inertial mass.

In the Hamiltonian formulation, adequate for Dirac's quantization, $p$ and $q$ are symmetrical. Fixing a Lagrange subspace, i.e. defining a genuine configuration space leads to an isomorphism with the cotangent bundle, and via Legendre transform to the Lagrangian Theory on the tangent bundle. Now mass is functional (see Wiki: tautological form).

So in principle, it does not have to be invariant...In what precise way it can be manipulated, remains to be investigated.

\section{Conclusions}

Gravity is a residual force, with a potential that may be modeled as thermal in origin (Alzofon, Verlinde's entropic Gravity, etc.), due to a chaotic nuclear orientation and anisotropy (L.I.).

This is at the "electric" (force capable of work) level; at the "magnetic level" (vector potential), it is capable of an induction effect, a "relativistic correction", yet experimentally significant, and demonstrable in the Lab.

Further questions need to be addressed.

\subsection{Mass and Quantum Levels}

Mass is an analog of impedance, with a "loss of heat" due to the "resistance" analog in an electronic circuit (except for being an $S U(2)$ analog). Impedance in two-body interaction channels can be controlled; hence Gravity can. How this fits in the Higgs model remains to be seen.

The mass quantum levels and proper decay times fit on $\alpha$-power scale [17] [18], which should be derived from the above mass model. How this extends the SM, Gell-Man-Nishijima and Okubo formulas, etc. remains to be seen.

\subsection{Fine Structure Constant}

This remains to be related to the only really fundamental mathematical structure that lies at the root of all (String models, Platonic models, etc.): the POSet of Prime numbers as the shadow of the Abelian category of $Z$-modules and their symmetries, together captured as the primary finite fields $F_{p}$ and their deformation theory ( $p$-adic analysis). See Michael Atiyah's recent "hints"; also [17].

Pauli's dream and Feynman's legacy will finally join into a unified picture of "Physics": It's just Math! This will justify Feynman's joke, Einstein's claim about how God thinks, etc., and finally reveal the Nature of "Nature".

Thus presently "we" are technically ready to unify "fundamental" interactions and ready to control Gravity at an engineering level...but are "we" in position to handle the consequences!? 


\section{Conflicts of Interest}

The author declares no conflicts of interest regarding the publication of this paper.

\section{References}

[1] Ionescu, L.M. (2007) In Search for a Unifying Principle. https://arxiv.org/abs/0705.1116

[2] Ionescu, L.M. (2018) The Platonic Universe and ADE-Correspondence. ISU Algebra Seminar. https://my.ilstu.edu/ lmiones/

[3] Zenczykowski, P. (2013) Elementary Particles and Emergent Phase Space. World Scientific. https://www.worldscientific.com/worldscibooks/10.1142/8918

[4] Ionescu, L.M. (2019) Qubit Model: A Platonic and Exceptional Universe. http://my.ilstu.edu/ lmiones/2019

[5] Ionescu, L.M. (2005) The Digital World Theory: An Invitation. Olymp Press, Romania. http://my.ilstu.edu/ lmiones/DWTv1.pdf

[6] Ionescu, L.M. (2017) A Holistic Approach to Quantum Physics. AASCIT Communications, 4, 19-26.

http://www.aascit.org/journal/archive2?journalId=940\&paperId=5323

[7] Ionescu, L.M. (2009) Infotronics: Theory and Experiment. http://my.ilstu.edu/ Imiones/ResearchProjects_2010.htm

[8] Ionescu, L.M. (2020) Quantum Gravity and Gravity Control. Tesla Extraordinary Conference Talk, 12 August 2021, Albuquerque, NM. http://www.teslatech.info/ttevents/prgframe.htm

[9] Matos, C.J. and Tajmar, M. (2000) Gravitomagnetic Barnett Effect. https://arxiv.org/ftp/gr-qc/papers/0012/0012091.pdf

[10] Ionescu, L.M. (2020) Prospects of a Unified Field Theory Including Gravity. https://vixra.org/abs/2104.0156

[11] Alzofon, F. (1981) Anti-Gravity with Present Technology: Implementation and Theoretical Foundation. 17th Joint Propulsion Conference, Colorado Springs, CO, 27-29 July 1981, 34 p. https://doi.org/10.2514/6.1981-1608

[12] Alzofon, F. (1994) The Alzofon Papers: Gravity Control. Electric Spacecraft Journal, \#13, 40 p. https://caylibrary.com/collections/journals/products/copy-of-esj-13

[13] Jeffries, C.D. (1963) Dynamic Nuclear Orientation. Interscience Publishers, New York.

[14] Bleaney, B. and Lounasmaa, O.V. (2003) Nuclear Orientation and Nuclear Cooling Experiments in Oxford and Helsinki. Part 2. Progress from 1945 to 1970. Notes and Records of the Royal Society of London, 57, 323-330. https://doi.org/10.1098/rsnr.2003.0218 https://www.jstor.org/stable/3557722? seq=1

[15] Edwards, J.C. (2009) Principles of Nuclear Magnetic Resonance. https://www.researchgate.net/publication/268026544_Principles_of_NMR

[16] Griot, M. (n.d.) Basic Laser Principles. Introduction to Laser Technology. https://www.bgu.ac.il/ glevi/website/Guides/Lasers.pdf

[17] Ionescu, L.M. (2019) e, $\pi, \chi \ldots \alpha$ ? https://vixra.org/abs/1912.0360

[18] Mac Gregor, M.H. (2007) The Power of $\alpha$ : Electron Elementary Particle Generation With $\alpha$-Quantized Lifetimes and Masses. World Scientific Publishing Company. 